\title{
Problems of Use in Industrial Design Products
}

\author{
Fekry Gamal Ibrahim ${ }^{1}$, AbdEInaby Abo EImagd ${ }^{1}$, Tamer Yousif ${ }^{2}$ \\ ${ }^{1}$ Industrial Design Department, Helwan University, Cairo, Egypt \\ ${ }^{2}$ Canadian International College for Engineering (CIC), Cairo, Egypt
}

Email address:

Tameryousif10@yahoo.com (T. Yousif)

To cite this article:

Fekry Gamal Ibrahim, AbdElnaby Abo Elmagd, Tamer Yousif. Problems of Use in Industrial Design Products. American Journal of Mechanics and Applications. Vol. 5, No. 3, 2017, pp. 22-27. doi: 10.11648/j.ajma.20170503.12

Received: May 25, 2017; Accepted: August 8, 2017; Published: October 6, 2017

\begin{abstract}
The ease of use becomes one of the most important factors that are seen with great interest from industrial organizations, designers, and takes a great deal of research and studies. It has become one of the basic pillars upon which to build the design and development policies, so as to reach the distinct relationship between the user and the product. This interest has increased as a result of the enormous technological development, the variety of industrial products and strong competition between companies to get a larger percentage of sales in the international markets, especially as we are in the era of globalization and open markets, which leads to increase the number of users, and therefore the need for product can be used easily to match different cognitive, cultural, social, and educational.... users' levels worldwide.
\end{abstract}

Keywords: Use, Use Problems, Cognitive Abilities, User Satisfaction

\section{Introduction}

Lately, interest in function and the technology to fulfill this function has been increasing at the expense of a user's culture, how the user understands such a technology and to what extent the user can deal with the product and use it easily. For example, some users cannot fully understand how to operate CD-DVD players, use video devices, digital cameras and TVs. These devices, with the functions they contain, often require long hours to understand them, whether through trial or reading User Manual countless pages.

Due to the difficulties and problems some users encounter in using some products and how to get the desired function, forcing them to waste much time and effort to figure out how to operate, use or perform some simple maintenance tasks. It may lead to users' reluctance to buy or use the product, meaning that the organization might lose potential profits.

\section{Process of Using a Product}

Use is an expression to describe a certain part of products life cycle. Generally, use indicates the user-productenvironment interaction. Recently, due to the need to significantly identify the nature of interaction between the human user and various products, use process researches have been increased.

Use process is done through the relationship and interaction between the user and the use environment components that include products, workspace, sequence of operations, the required task and organizing and interacting among these factors. Therefore the product should fit the user, consistent with his abilities, his personality traits, his social status, etc, along with being surrounded with proper use environment that enables it to perform capably and effectively. The user's performance of use process in a convenient manner makes him feel satisfied and happy.

\subsection{Use as a Process}

A process can be defined as "a series of actions, a natural or automatic operation or a series of changes". As Cain believes [9] use process is built through process details, as it represents:

1. Control: representing the actions made by user to affect product function,

2. Display: displaying product information to user, and

3. Final Results: expressing product function.

\subsection{Problems of Use in Industrial Design Products}

Users often encounter problems when dealing with hi-tech products and trying to utilize all their various advantages. 
Industrial companies do not pay attention to users' mental abilities. Problem of use of hi-tech products caused a wide gap between users and such products. Users find themselves unable to use products and get the benefit, Figure 1.

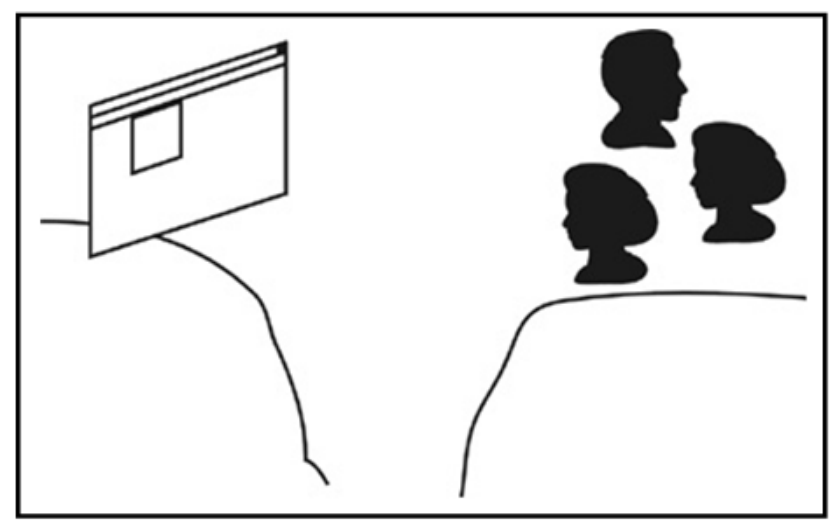

Figure 1. The gap between users and hi-tech products.

This problem cannot be solved by product micro display means and microcontrollers. Interfacing with user must be designed so as to help the user on how to use, especially with the complicated features and additions. Interfacing must work on providing the user with a mechanism that enables him to answer questions: who, how, when and why. These are the questions the user encounters. The user has to refer to the User Manual, contact the helpline or is forced to withdraw.

To know the reasons for considering these products misused are as follows:

\subsubsection{Ignorance of Users}

Many manufacturers of hi-tech products do not have good knowledge about users. Most of these products are designed without a good understanding of users, why they need a product, why they choose a certain product from competitive products, and how they will use the products. Companies must exert more effort to know the users closely in terms of their cultural, social, educational, technological, etc experience levels. There should be a significant cooperation between manufacturing companies and the companies specialized in user's research field.

\subsubsection{Conflict of Interest Between Meeting User's Needs and Fulfilling the Features}

This conflict is due to those in charge of designing such products. They are also the persons in charge of these companies and their investments by having consulting design offices. Hence, they are required to choose between achieving ease of use and achieving competitive advantages to their companies by adding technology. It is inappropriate to forget that technology is an interesting matter for users and designers. For designers, it is easy to add attractive features. For users, they can feel they are citizens about whom no one cares unless their products have the latest features. Even when adequate skills and good intentions are combined, it is not easy to defend the user, industrial enterprise and technology at the same time. Finally, the designer surrenders to investment pressures, favoring the factor of adding technology to user's abilities factor.

\subsubsection{Lack of Processes}

This means lack of fully accredited and systematic processes that a designer follows to make hi-tech products easy to use. Thus, engineering departments should follow accurate and strict methods to ensure and assure the feasibility of technology.

Assisting departments, like marketing and sales departments, should make efforts to analyze to what extent users understand these new products. Designers finish their design of user interface in the same manner as miners finish their design of drilling scheme. Hence, users suffer inability to understand it. Unfortunately, the design processes at noninterested companies and development organizations interchange between casual and nonexistent methods.

\section{Impact of Use Problems on User's Cognitive and Economic Aspects}

\subsection{Impact of Use Problems on User's Cognitive Aspect}

When a designer designs incomprehensible controllers or display means which ideal use is unclear, it leads to the user's confusion, forcing him to think a lot about dealing and use. It is noted that many of the errors made by some users when dealing with many products, especially hi-tech (digital) products, that requires persons who think like computers. Such products always assume that the users are technologyliterate, that they will change their thinking to adapt with how this type of products works.

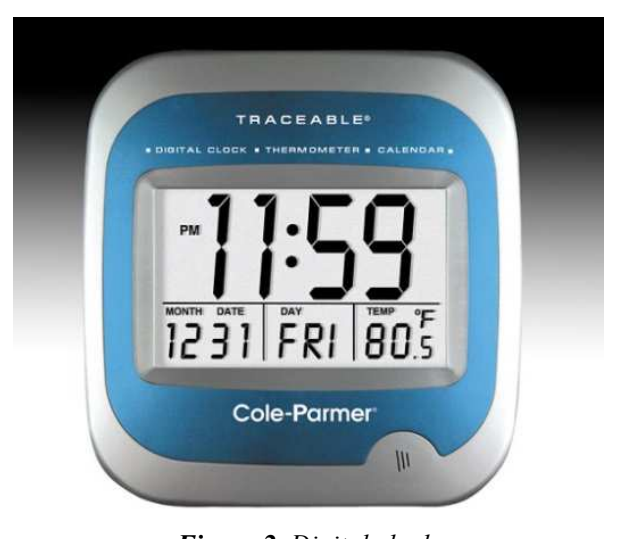

Figure 2. Digital clock.

Errors the users might make due to misconception can be divided into 3 categories:

1. Perception Errors: a user misperceives how a product performs and how to utilize the product due to unavailability of the required information and unknown codes, incompatibility with the user's mental model or having incomprehensible procedures.

2. Decision Errors: a user does not respond to an indication or information due to thinking it is unimportant or not a priority. On the other hand, the 
user may misjudge the situation, due to poor interface planning or excess of information and difficulty in exploring the interface.

3. Action Errors: a user responses to the effect, but user chooses a wrong controller, or deals with the correct controller erroneously due to inconvenient modes, difficulty in setting processes, many identical tasks or lack of reaction in case of error.

As a practical example to these various types of errors, a product (a digital clock), Figure 2, was tested.

The test results indicated that the different types of errors because of misperception are as in Table 1:

Table 1. shows the resulted errors of the Digital Clock test.

\begin{tabular}{lll}
\hline Perception Errors & Decision Errors & Doing Errors \\
\hline - Difficulty in understanding display tools. & - Difficulty in reading letters. & - Difficulty in pressing the control as it is too \\
& & small. \\
- Difficulty in doing some processes. & - Difficulty in seeing display means. & - Difficulty in pressing the controls because the \\
- Some controls cause confusion because they are & & distances between them are too small. \\
linked to several functions. & & \\
\hline
\end{tabular}

To speak in details about why users feel confused, the paper must refer to the mistake that some industrial companies do: persisting on Implementation Model to design the product. It is a model of how programs operate and how users receive the functions they need to do. This model reflects technology without conformity with the Mental Model, a model that is based on users' ideas about how to perform functions, how the devices work and how the program help them to do so. Designers create a picture or model in their brains about how the things work. So, they suggest blaming the user as the direct reason for any problem is the user because he cannot understand strange things and complicated mechanism.

However, users do not need to know the details of how complicated mechanism works so they can use it. For example, many people imagine that when they have the wire of vacuum cleaner plugged into a power supply in the wall, the electricity flow becomes like water flow; it flows from the power supply in the wall to the device/ tool through the electric wire. This Mental Model is completely suitable for home appliances use. There is another model in the digital products world called Represented Model. It is a way by which a designer chooses to offer the functions to the user. Figure 3 shows these three models.

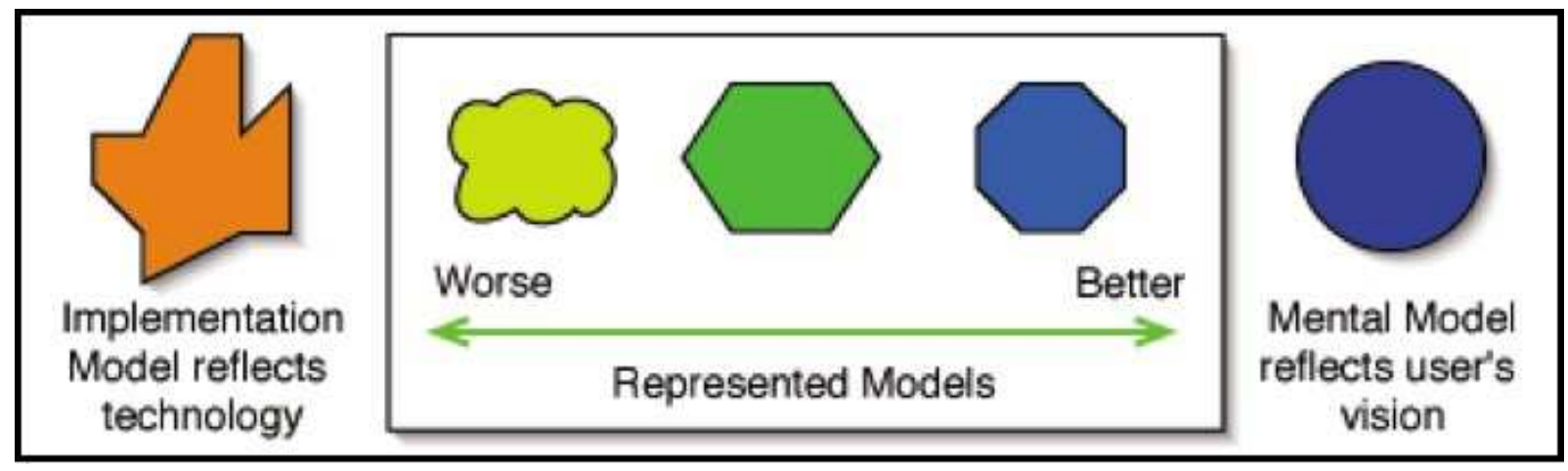

Figure 3. The three models of designing a product.

\subsection{The Effect of User Problems on the Economical Aspect of the User}

Some industrial companies focus more on technology, not on users by respecting their mental abilities. This resulted in the users' inability to utilize and get some advantages and functions that a product includes. This may frustrate the user. Furthermore, these problems reflected negatively on the economical aspect of the user. The user found himself paying a lot of money to buy a product, but user does not benefit from all this money because there are some functions in the product he cannot use, or because there are too many functions in the product he does not need at all.

For example, the user may pay thousands of pounds to buy a mobile phone because of a feature or some features he needs, but user does not benefit from the other features in the product because user cannot use or does not need at all. In this case, the user pays a lot of money for little benefit.

Thus, many industrial companies working in the field of electronic devices noticed that the products that had the lowest sales and were most rejected by users were at the same time the products that had the highest technology and multiple advantages. Such products are those that the user insists they must be easy to use and their use is of paramount importance, Figure 4.

As for the negative effect on the economic side of companies, users return many products to the companies because they cannot use all the advantages provided with products and it is difficult to use such products. Consequently, the companies have a critical situation forcing them to pay a lot of money for redeveloping their products, to produce products that overcome the use difficulties that such products have and to maintain their market share. 
Table 2 shows samples of product returns to (Philips).

Table 2. Samples of returns of Philips.

\begin{tabular}{lllllll}
\hline \multicolumn{2}{|c}{ April 2001 - May 2002 } & & \multicolumn{3}{l}{ April 2002 - May 2003 } \\
\hline Distributor & Total products sold & $\begin{array}{l}\text { Number of } \\
\text { returns }\end{array}$ & $\begin{array}{l}\text { Percentage of } \\
\text { returns }\end{array}$ & Total products sold & $\begin{array}{l}\text { Number of } \\
\text { returns }\end{array}$ & $\begin{array}{l}\text { Percentage of } \\
\text { returns }\end{array}$ \\
\hline $\mathrm{ABC}$ & 768.74 & 49.276 & $6.41 \%$ & 596.73 & 35.028 & $5.87 \%$ \\
\hline
\end{tabular}

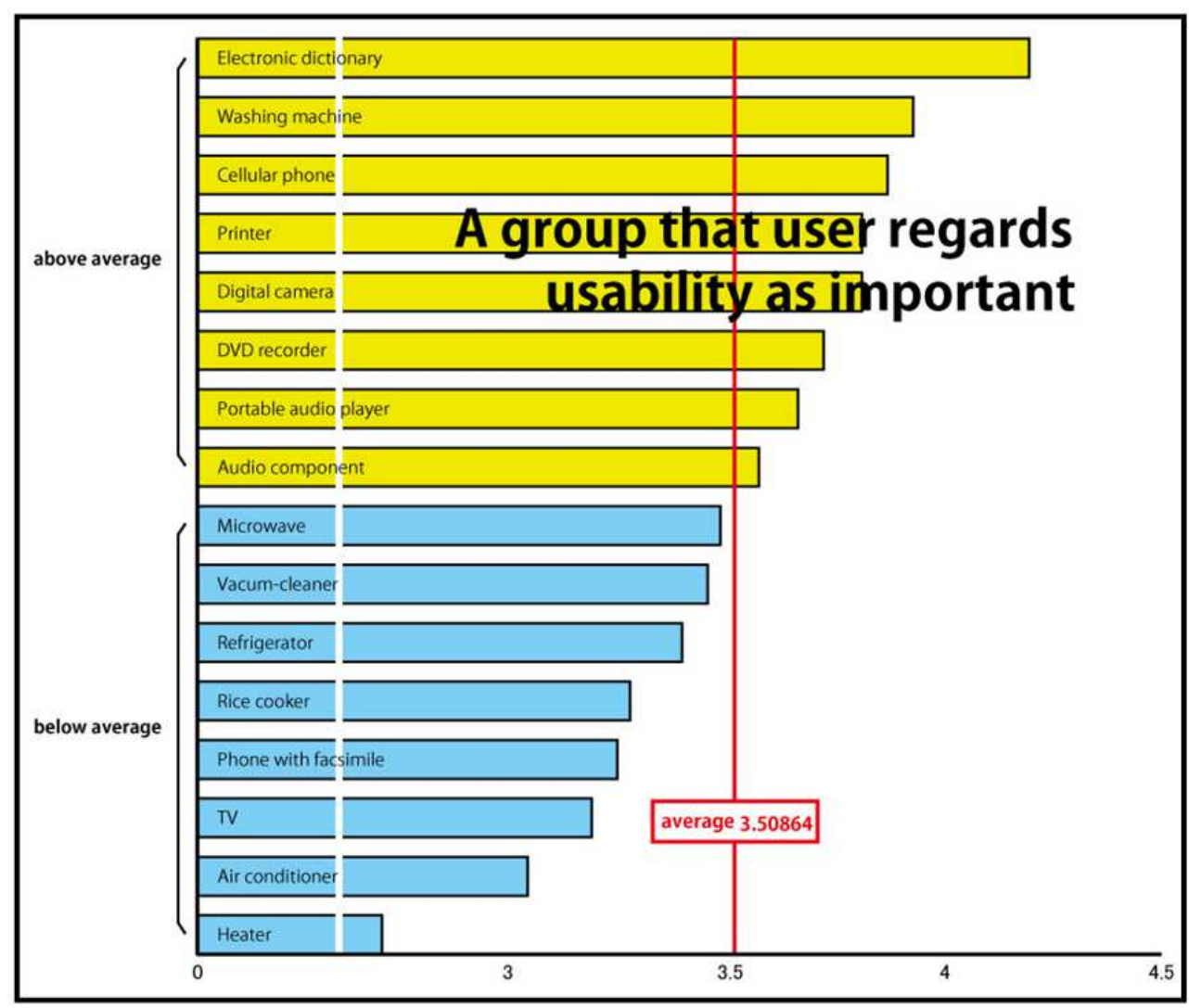

Figure 4. Top electronic products that need ease of use.

\section{Practical Examples of Use Problems of Some Industrial Design Products}

In their daily lives, people use many products to meet their needs and utilities. Many of these products are not easy to use and one cannot know a function easily. Therefore, one faces a lot of problems and difficulties of use when dealing with many different daily life products.

Table 3 shows practical examples to the problems of use of some different simple products, home appliances and electronic devices.

Table 3. Examples of usage problems in industrial design products.

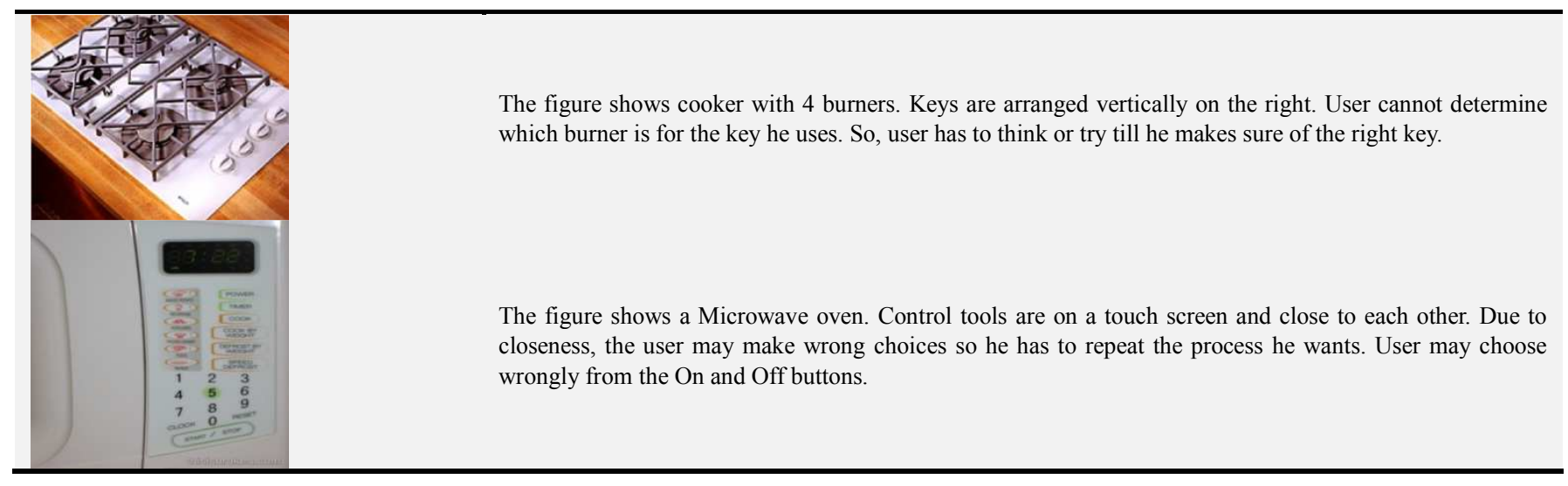




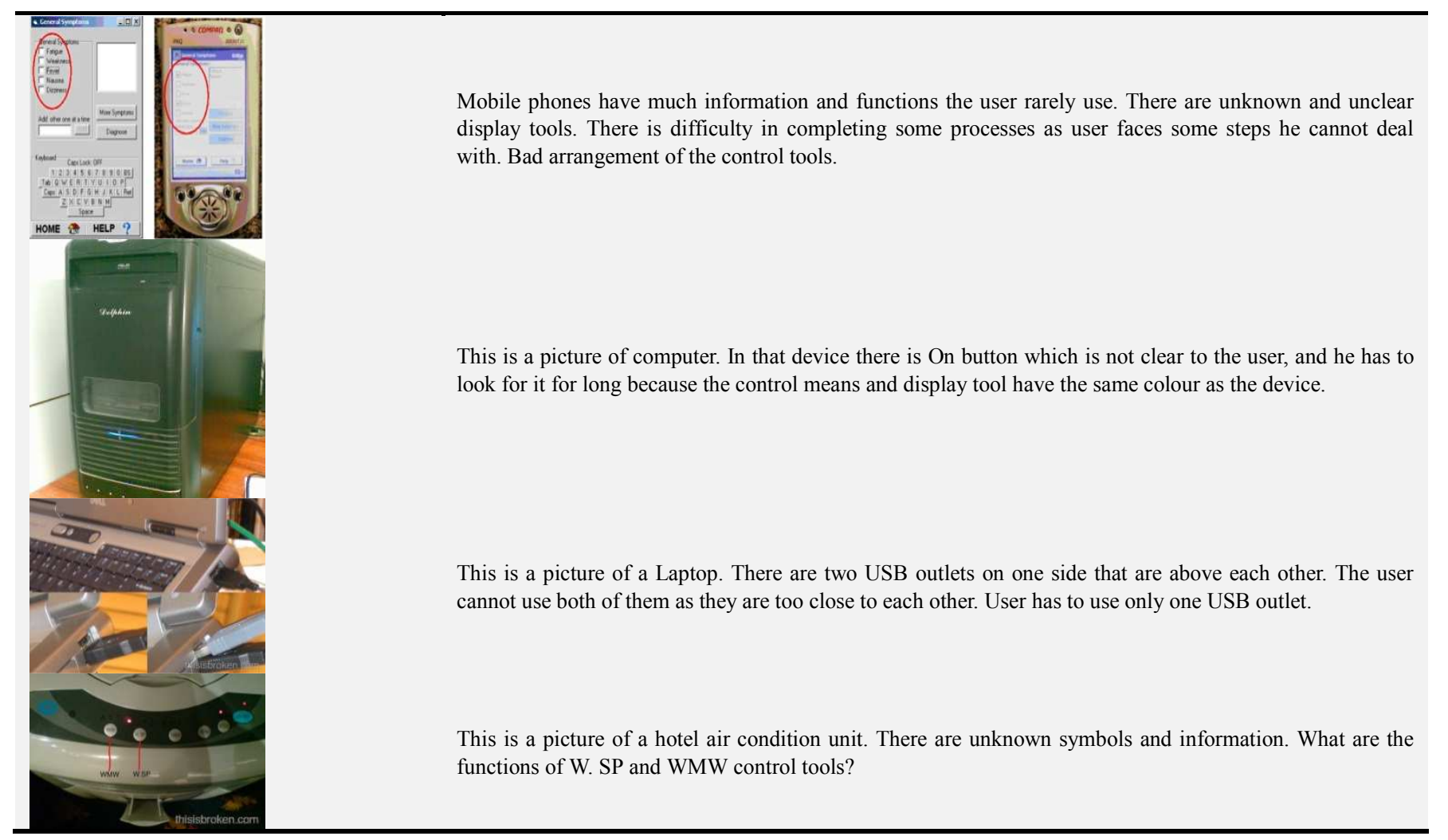

\section{Industrial Designer's Role in Solving the Use Problems}

The role of industrial designer is as follows:

1. Designing a product/ system that a user can understand.
Communicating between the displayed model and the user mental model as close as possible. So, it is important for designers to understand the way users think towards the job they are doing and to understand their beliefs about how the things work. Figure 5.

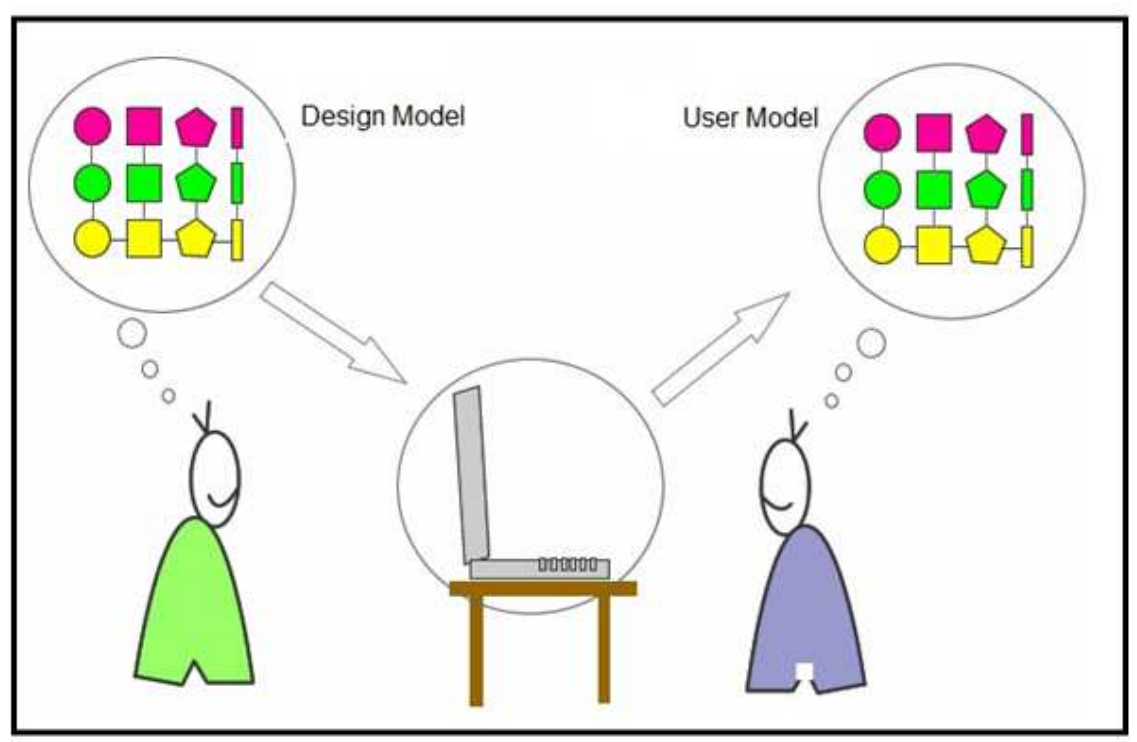

Figure 5. The importance of communication between the displayed and mental models.

It should be noted that the mental model is often true, and the way a product works functions is as expected by the user. In other times, it is wrong, in which case the user finds it difficult to use the product.
There are also several challenges a designer faces during designing a product, like:

1. Designing an interface that can be understood by two different users. 
2. Arranging and providing information in a meaningful way and in the form of logical orders.

Figure 6 shows a group of those challenges.

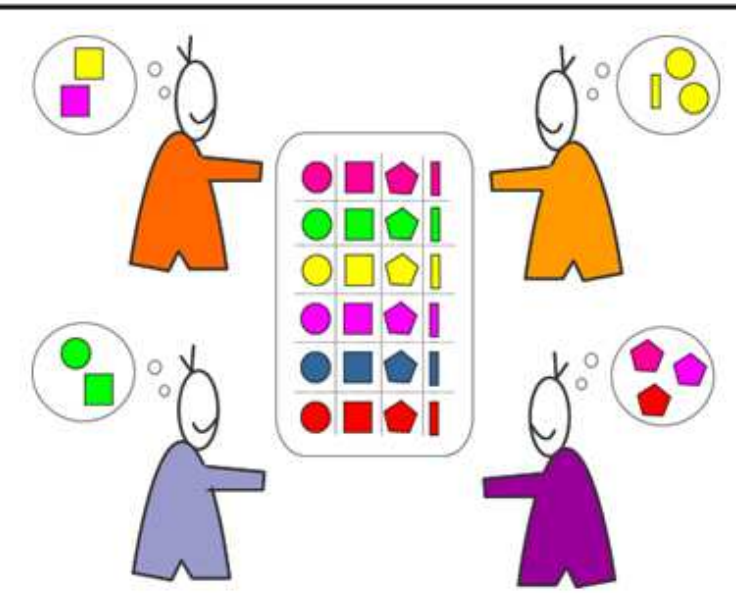

The product has different users with different goals
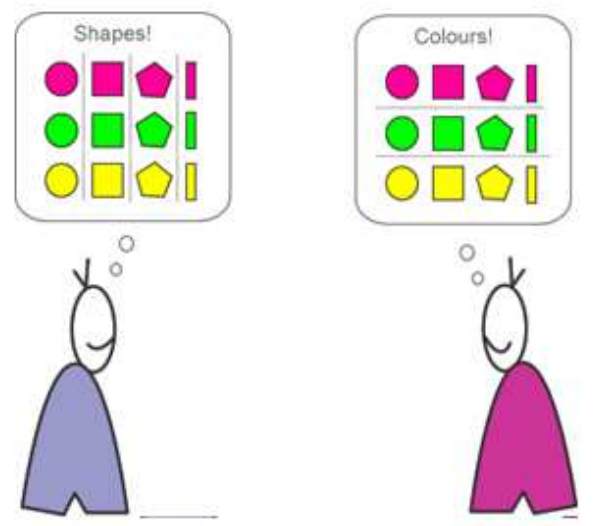

Good design should allow different users to access content in different ways

Figure 6. Challenges group facing the designer.

\section{Results}

1. The need to design use elements of the product for ease of use.

2. Some products are not easy to use and the user is frustrated because he cannot get the benefit.

3. The designer should follow a methodology to ensure design products easy to use and achieve the satisfaction of the user.

4. Emphasize the study of the user sufficiently from all aspects and make him the centre of the design process to get products that achieve satisfaction.

5. Not only focus on the product's functions, technological advantages of the product, and neglect the capabilities of the user so that he can understand those features.

\section{Conclusion}

The product must be usable without any complications that will make the users in a state of confusion using the product, if the users do not understand something, they will not use it. The users should access to product's functions without much mental effort.

\section{References}

[1] Abdul Nabi Abul Magd: Ergonomics in Industrial Design, Giza, 2000.

[2] Alon Cooper, Robert Reimann and Dave Cropin: About Face 3: the Essentials of Interaction design, Wiley Publishing, USA, 2007.

[3] Chang-franw lee, Che-cheng liao: Study of the product usability for elderly Taiwanese people, school of Industrial design, National Yunlin University, Taiwan.

[4] Dey Alexander: An Introduction to usability, faculty of Art \& Design, Monash University, 2004.

[5] George M. Donahue, Susan Weinschenk, Julie Nowicki, Usability Is good business, compuware, USA, 1999.

[6] Helander, Martin: A Guide to the ergonomics of manufacturing, Taylor \& Francis Ltd., London, 1995.

[7] J. I. Van KuiJK, H. H. C. M. Christiaans, H. Kanis, D. J. Van EiJk, Usability in product development, A Conceptual Framework, Faculty of Industrial design engineering, the Netherlands, 2001.

[8] Ravi Kiran Vatrropu; Culture and international usability testing, The effects of Culture in interviews, MSC, Virginia university, USA, 2002.

[9] Wilhem F. (Wilfred) van der vegte \& Imre Horath: Consideration and modeling of use processes in computer Aided Conceptual design: A state of the Art review, Journal of Integrated design and process science, USA, June 2002, vol. 6, No. 2.

[10] William S. Green and Patrick w. Jordan, Pleasure with products: beyond usability, Taylor \& Francis Ltd., New York, 2005. 